\title{
RESET
}

Recherches en sciences sociales sur Internet

\section{Les pratiques des écrans des jeunes français}

Déterminants sociaux et pratiques culturelles associées

French Youth and Screens. Cultural Practices and their Social Determinants

Fabienne Gire et Fabien Granjon

\section{CpenEdition}

Journals

Édition électronique

URL : http://journals.openedition.org/reset/132

DOI : 10.4000/reset.132

ISSN : 2264-6221

Éditeur

Association Recherches en sciences sociales sur Internet

Référence électronique

Fabienne Gire et Fabien Granjon, « Les pratiques des écrans des jeunes français », RESET [En ligne],

1 | 2012, mis en ligne le 30 décembre 2012, consulté le 01 mai 2019. URL : http://

journals.openedition.org/reset/132; DOI : 10.4000/reset.132

Ce document a été généré automatiquement le 1 mai 2019.

(c) Association Recherches en sciences sociales sur Internet 


\title{
Les pratiques des écrans des jeunes français
}

\author{
Déterminants sociaux et pratiques culturelles associées \\ French Youth and Screens. Cultural Practices and their Social Determinants
}

\author{
Fabienne Gire et Fabien Granjon
}

Il semble aujourd'hui convenu de reconnaître combien les technologies numériques modifient notre rapport à la culture dans ses dimensions pratiques comme symboliques. Après la massification de la culture, notamment liée au développement des médias audiovisuels et des industries culturelles, le numérique vient à son tour transformer les pratiques de production, de diffusion et de catégorisation des contenus culturels et des biens d'expérience, lesquels deviennent pléthoriques, gratuits et non-rivaux. Ces déplacements «en amont » modifient aussi les habitudes des individus qui explorent de nouveaux cadres formels de consommation culturelle: délinéarisation, acquisition massive, fragmentation, mobilité, etc. (Donnat \& Lévy, 2007). Aussi est-il communément admis que le numérique s'immisce toujours davantage dans le quotidien des Français et devient une ressource culturelle majeure de la jeunesse. Si l'assertion n'est pas une contre-vérité à son plus haut niveau de généralité, dans la mesure où nombre des pratiques portées par les jeunes les distinguent effectivement de leurs aînés, il est toutefois indispensable de préciser que derrière ce qui est présenté comme la nouvelle culture numérique des jeunes se cachent in fine des culturèmes sensiblement différenciés (et différenciants), ainsi que des modalités variées d'appropriation. Celles-ci restent dépendantes des conditions sociales d'existence des jeunes, lesquelles n'ont rien d'homogène. Si force est de reconnaître que nombre de pratiques culturelles sont pour partie liées à l'âge (Pasquier, 2005 ; Gire, Pasquier \& Granjon, 2007 ; Octobre et al., 2010), il ne faut pas pour autant perdre de vue qu'à l'intérieur même de la population jeune, parcours sociaux, trajectoires d'usages et structuration des goûts ne sont pas, tant s'en faut, similaires. 
2 A prendre au sérieux que la «jeunesse n'est qu'un mot» (selon la formule de Pierre Bourdieu, 1984) et que la culture numérique est avant tout une catégorie du sens commun, il s'avère nécessaire de déconstruire ces notions par trop englobantes. Pour ce faire, il faut d'abord noter que la jeunesse comme catégorie statistique est l'objet de définitions fort variées. La dé-standardisation des étapes du cycle de vie et la réversibilité de certaines transitions conduisent notamment à la reconsidérer sous l'angle d'un «continuum très extensif» (Octobre, 2011a). Dans le cadre de cet article, nous ferons nôtre un découpage retenant une classe d'âge allant de 15 à 34 ans $^{1}$. En mobilisant les données de la dernière enquête Pratiques culturelles des Français ${ }^{2}$ réalisée par le Ministère de la Culture (EPCF 2008), nous proposons d'apporter un premier éclairage sur la morphologie des usages des écrans déployés par les jeunes. Dans un premier temps, nous analyserons la diversité de leurs pratiques, puis nous dégagerons les grandes figures d'appropriation qui structurent leurs consommations et chercherons à en identifier les principaux déterminants.

\section{De la diversité des pratiques des écrans chez les jeunes}

3 Nous proposons donc de nous intéresser aux pratiques des écrans des 15-34 ans, en nous appuyant sur une analyse de correspondances multiples (ACM). Celle-ci a été effectuée sur les variables de fréquences des pratiques (regarder la télévision, des vidéos, jouer à des jeux vidéo, utiliser l'ordinateur et Internet, ainsi que deux variables traduisant l'attachement à la télévision) et le temps passé devant les trois principaux écrans (téléviseur, ordinateur et console de jeux). Elle nous permet de faire apparaître une première série de proximités et de dissemblances entre les différents usages des écrans mobilisés par les jeunes. Les variables prises en compte dans l'analyse sont présentées dans le tableau 1.

4 L'étude de l'histogramme des valeurs propres nous invite à décrire les quatre premiers axes factoriels représentant près de $22 \%$ de l'inertie totale (selon les critères de la contribution moyenne, du coude et du scree test de Catell). Les deux premiers axes factoriels marquent une opposition très nette entre les utilisateurs et les non-utilisateurs de la télévision (axe 1) et de l'ordinateur (axe 2)3, tandis que les deux suivants permettent de graduer l'intensité des pratiques numériques (télévision, vidéos, jeux vidéo pour l'axe 3 et ordinateur/Internet pour l'axe 4).

Tableau $1:$ Variables actives de l'ACM

\begin{tabular}{|l|l|l|}
\hline \multirow{4}{*}{ Télévision } & $\begin{array}{l}\text { Regardez-vous la télévision, que ce soit chez vous ou ailleurs, chez des } \\
\text { parents, des amis, au café, etc. ? (fréquence) }\end{array}$ & Q27* \\
\cline { 2 - 4 } & $\begin{array}{l}\text { Vous arrive-t-il d'allumer la télévision en rentrant chez vous, sans } \\
\text { connaître le programme? }\end{array}$ & Q28 \\
\cline { 2 - 4 } & $\begin{array}{l}\text { Si vous ne pouviez plus regarder la télévision pendant 2 mois, pensez- } \\
\text { vous que cela vous manquerait? }\end{array}$ & Q35 \\
\cline { 2 - 4 }
\end{tabular}




\begin{tabular}{|c|c|c|}
\hline & $\begin{array}{l}\text { En moyenne, combien de temps regardez-vous la télévision (sans } \\
\text { compter émissions enregistrées ou téléchargées) }\end{array}$ & Q36A \\
\hline \multirow{2}{*}{ Vidéo-DVD } & $\begin{array}{l}\text { Au cours des } 12 \text { derniers mois, tous les combiens, en moyenne, avez- } \\
\text { vous regardé des DVD ou des vidéos cassettes, que vous les ayez } \\
\text { achetées, louées, enregistrées ou téléchargées? }\end{array}$ & Q39 \\
\hline & $\begin{array}{l}\text { En moyenne, combien de temps utilisez-vous personnellement la } \\
\text { télévision pour regarder des DVD ou des cassettes vidéo? }\end{array}$ & $\begin{array}{l}\text { Q40A } \\
\text { Q40B }\end{array}$ \\
\hline \multirow{2}{*}{ Jeux vidéo } & $\begin{array}{l}\text { En général, à quelle fréquence jouez-vous à des jeux vidéo (que ce soit } \\
\text { sur une console de jeux, un téléphone portable, un écran de télé ou un } \\
\text { ordinateur)? }\end{array}$ & Q13 \\
\hline & $\begin{array}{l}\text { Personnellement, combien de temps passez-vous à jouer à des jeux } \\
\text { vidéo? [à tous les joueurs, sauf ceux qui jouent le plus souvent sur un } \\
\text { micro] }\end{array}$ & Q16 \\
\hline \multirow{3}{*}{$\begin{array}{l}\text { Ordinateur- } \\
\text { Internet }\end{array}$} & $\begin{array}{l}\text { Personnellement, à quelle fréquence utilisez-vous un ordinateur chez } \\
\text { vous ou ailleurs, toujours sans compter les utilisations } \\
\text { professionnelles? }\end{array}$ & Q19 \\
\hline & $\begin{array}{l}\text { Personnellement, au cours des } 12 \text { derniers mois, à quelle fréquence } \\
\text { vous êtes-vous connecté à Internet, quels que soient le lieu et le mode } \\
\text { de connexion? }\end{array}$ & Q22 \\
\hline & $\begin{array}{l}\text { Finalement au total, combien de temps estimez-vous passer en } \\
\text { moyenne devant l'écran de votre ordinateur à des fins personnelles } \\
\text { (Internet compris et quel que soit le lieu pour les portables)? }\end{array}$ & Q26 \\
\hline
\end{tabular}
* Renvoie au numéro de la question. Pour le détail des modalités proposées :
www.pratiquesculturelles.culture.gouv.fr/doc/08questionnaire.pdf

Le premier axe factoriel oppose ainsi les téléspectateurs aux individus ne regardant jamais ou presque jamais le petit écran, opposition qui s'avère liée aux caractéristiques sociodémographiques des interviewés ainsi qu'à leurs pratiques culturelles et de loisirs. Les 15-34 ans qui ne regardent jamais ou presque jamais la télévision épousent plutôt les caractéristiques des populations les plus socialement et culturellement favorisées : très diplômés (un niveau Bac+4 et plus), encore étudiants ou bien occupant un poste de cadre s'ils travaillent, célibataires sans enfant, vivant dans Paris intra-muros et issus d'un milieu favorisé (père cadre, au moins un parent diplômé du supérieur). En revanche, le fait de ne pas regarder la télévision semble peu lié au genre et au capital économique (estimé par le revenu par unité de consommation afin de tenir compte de la taille du foyer), mais se voit associé à une pratique importante de lecture de livres, d'écoute de musique et de sorties et à l'absence d'écoute de la radio. Notons que si le non-usage de la télévision est de facto plus répandu parmi les populations les plus socialement et culturellement favorisées, il demeure néanmoins une non-pratique largement minoritaire. Particulièrement répandue, la consommation télévisuelle se présente comme une pratique culturelle qui n'est, en soi, ni stigmatisante, ni distinctive. Aussi, ce sont davantage les contenus mobilisés, ainsi que les manières de consommer qui sont 
susceptibles de réintroduire des régimes de rareté à partir desquels des formes de distinction viennent éventuellement se greffer (Granjon, Combes \& Ville, 2009).

6 Le deuxième axe oppose les non-utilisateurs de l'ordinateur aux usagers réguliers (principalement quotidiens) de l'informatique. La plupart des modalités sociodémographiques testées en variables illustratives présentent des valeurs test supérieures à 2 en valeur absolue, ce qui traduit une forte liaison entre l'usage de l'ordinateur et les caractéristiques sociodémographiques de l'individu. Le non-usage de l'informatique est ainsi plutôt le fait d'individus présentant les caractéristiques des classes populaires (capital scolaire faible, emploi peu qualifié ou sans emploi, origine sociale modeste, capital économique peu élevé, habitant des communes rurales ou de petites agglomérations, etc.). Il est aussi davantage le fait de la tranche la plus âgée des jeunes (les 24-35 ans) et de ceux qui élèvent des enfants. Inversement, l'usage régulier est plutôt attribué aux individus disposant d'un capital scolaire élevé (diplômés du supérieur ou poursuivant des études), urbains, dotés d'un capital économique plus élevé, issus d'un milieu social plutôt favorisé et vivant chez leurs parents. Par ailleurs, si les oppositions en termes de genre sont moins marquées, elles n'en demeurent pas moins significatives, l'usage régulier de l'ordinateur étant davantage associé aux hommes et le non-usage aux femmes. En ce qui concerne les pratiques culturelles, les exclus du monde informatique sont aussi les exclus des sorties. À l'absence (ou quasi-absence) de sorties, s'ajoute la non lecture de livres et le faible investissement dans l'écoute de musique. Inversement, l'usage régulier de l'informatique est associé à des sorties fréquentes, à une écoute quotidienne de musique et à une consommation de livres modérée à forte ${ }^{4}$. Par ailleurs, contrairement à la consommation télévisuelle, les usages de l'informatique apparaissent comme plus discriminants (moins rares) et le "non-usage " possède tous les atours d'un phénomène de polarisation sociale (Granjon, 2010). Au sein des classes populaires, quand les niveaux d'utilisation rejoignent ceux des classes supérieures, les contenus consommés et les « arts de faire » tendent également à coïncider, notamment parce qu'ils sont, au sein des classes populaires, le fait quasi exclusif de "dominés aux études longues " (Schwartz, 2002), lesquels, par leur capital culturel, se rapprochent, sous cet aspect, des individus des classes supérieures, en partageant notamment leurs dispositions culturelles.

7 Le troisième axe factoriel oppose les forts consommateurs de télévision, vidéos et jeux vidéo aux faibles consommateurs qui regardent certes la télévision, mais qui n'y sont pas pour autant attachés (leur consommation n'est pas quotidienne, ils ne l'allument pas en rentrant chez eux et affirment qu'ils ne ressentiraient pas de manque s'ils en étaient privés), qui ne visionnent pas de vidéos ou rarement et qui ne jouent pas aux jeux vidéo. La faible consommation est davantage le fait d'individus présentant un capital scolaire élevé, issus d'un milieu social favorisé, urbains, tandis que la forte consommation est surtout associée aux caractéristiques des classes populaires. L'âge et le genre sont également discriminants, la faible consommation étant plutôt associée aux 25-34 ans et aux femmes, tandis que la forte consommation relève davantage des 15-17 ans, des 18-24 ans et des hommes. On notera par ailleurs que la situation familiale n'est pas neutre, les individus élevant des enfants seuls ou en couple sont en effet associés à une faible consommation, peut-être par manque de temps disponible. En revanche, le niveau de vie ne semble pas lié à l'intensité cumulée de consommation de télévision, de vidéos et de jeux vidéo. En matière de pratiques culturelles, les oppositions sont moins nettes et plus difficiles à interpréter que sur les deux axes précédents. Une faible consommation de 
télévision, de vidéos et de jeux vidéo est davantage couplée à une faible écoute de radio et de musique, tandis qu'une forte consommation s'adjoint plutôt à une durée d'écoute de la radio élevée et à l'écoute quotidienne de musique. En revanche, on ne note pas de différences significatives en termes d'intensité de lecture de livres. L'absence de sorties est davantage liée au faible usage cumulé TV-vidéos-jeux vidéo. Mais il s'agit d'une situation extrêmement peu répandue parmi les 15-34 ans, puisqu'elle concerne seulement $4 \%$ d'entre eux. L'univers télévisuel et vidéo(ludique) donne donc matière au développement d'usages qui semblent, pour partie, se construire sur des clivages assez similaires, du point de vue de la stratification sociale, à ceux qui caractérisent la consommation strictement télévisuelle (axe 1). Toutefois, les couplages aux autres types de pratiques culturelles révèlent des configurations à la fois plus complexes, car travaillées par l'âge et le genre, mais également plus marquées, qui doivent être le fait d'individus ayant des profils particulièrement contrastés au regard du rapport qu'ils entretiennent à la culture audiovisuelle.

Enfin, le quatrième axe factoriel oppose les gros utilisateurs de l'ordinateur et d'Internet (usagers quotidiens et consacrant plus de 15 heures à cette activité par semaine) aux utilisateurs plus modérés. Cet axe est principalement illustré par le genre, les pratiques intenses étant plutôt masculines et les pratiques modérées plutôt féminines. En termes de pratiques culturelles, les contrastes sont sensiblement moins nets et portent uniquement sur l'écoute de musique et la fréquence de sorties. Ainsi, la pratique intense de l'informatique connectée est associée à une écoute quotidienne de musique et à l'absence de sorties ${ }^{5}$. Inversement, l'écoute non quotidienne de musique est davantage associée à une pratique occasionnelle de l'ordinateur, lequel est devenu un système technique intégré et polyvalent à partir duquel on obtient, on centralise, on écoute et on redistribue des contenus musicaux, facilitant ainsi leur fréquentation (Granjon \& Combes, 2007).

La description des deux premiers axes factoriels révélés par l'ACM met donc en évidence les différences de positionnement du téléviseur et de l'ordinateur dans l'espace des loisirs des jeunes. Alors que ne pas regarder la télévision (situation toutefois marginale) est associé à des pratiques culturelles intenses, l'absence d'usage de l'ordinateur révèle au contraire un investissement réduit dans les sorties, la lecture ou l'écoute de musique, dessinant ainsi deux univers sensiblement distincts, l'un marqué par une logique de cumul, l'autre associé à une forme de " dénuement » culturel (Gire, Pasquier \& Granjon, 2007). Quand ceux qui délaissent la télévision sont plutôt issus des classes socialement et culturellement favorisées et investissent d'autres pratiques culturelles de façon conséquente (lecture, musique, sorties), les plus rétifs à l'informatique (connectée) se recrutent plutôt au sein des rangs des classes populaires et tendent à délaisser les pratiques qui sont précisément les plus courues par leurs pairs mieux dotés qu'eux. Une enquête que nous avions menée il y a quelques années (Granjon, 2009a) nous avait conduit à souligner que si les usages de l'informatique connectée au sein des classes populaires pouvaient être, parfois, tout à fait conséquents, ils étaient en ce cas, et pour l'essentiel, le fait d'individus assez fortement diplômés à la recherche de territoires numériques répondant à des intérêts culturels singuliers, lesquels n'étaient ni ceux des classes supérieurs, ni ceux de la fraction non-diplômée des classes populaires. Internet leur donnait alors les moyens de cet "entre-deux culturel», permettant, d'une part, d'effectuer des emprunts à une culture "consacrée », en levant certains des obstacles pratiques et symboliques qui en limitent habituellement l'accès, et d'autre part, de garder contact avec une culture « populaire » et médiatique dont ils allaient toutefois chercher à 
consommer les éléments les moins communs. Ces rapports hétérogènes à la culture étaient encore plus prégnants chez les $12-25$ ans qui affirmaient construire encore plus de ponts que leurs aînés entre leurs intérêts culturels singuliers et leurs pratiques d'Internet. Il n'est donc pas étonnant de constater que l'analyse du troisième axe révèle une logique de cumul des consommations de télévision, vidéo et jeux vidéo, davantage associée aux individus présentant les attributs des classes populaires, mais révèle également le rôle que jouent les variables d'âge, de sexe et de positionnement dans le cycle de vie (e.g. mise en couple, arrivée du premier enfant, etc.). Enfin, l'axe 4 fait une nouvelle fois apparaître les dissemblances de genre associées à l'intensité d'usage de l'informatique. Les jeunes hommes développent des répertoires de pratiques plus étoffés, alimentés à la fois par l'informatique connectée, l'univers vidéo-télévisuel et les jeux, et marqués par des investissements sensiblement plus conséquents que chez les jeunes femmes qui consacrent moins de temps aux loisirs sur écran. La prise en compte simultanée de ces quatre dimensions nous permet de construire une typologie des formes d'appropriation des écrans chez les jeunes et de revenir sur les effets respectifs de ces différents déterminants.

\section{Cinq formes d'appropriation des écrans chez les jeunes}

10 Afin d'obtenir une vision synthétique des différentes formes d'appropriation des écrans chez les jeunes, nous avons entrepris une classification ascendante hiérarchique (CAH) selon la méthode de Wald sur les quatre premiers facteurs de l'ACM précédemment présentés. Celle-ci nous invite à considérer cinq profils d'usagers des écrans. L'étude des variables illustratives (éléments sociodémographiques et fréquences des pratiques culturelles) permet d'interpréter les comportements.

\section{Les screenagers}

11 Le premier profil, le plus répandu, rassemble 39\% des 15-34 ans. Les screenagers constituent le groupe qui investit le plus la télévision et les nouveaux écrans. Télévision, ordinateur et Internet font effectivement partie de leur quotidien. Leur attachement au petit écran est particulièrement fort, puisqu'ils l'allument tous les jours ou presque en rentrant chez eux sans connaître nécessairement le programme et imaginent un manque s'ils en étaient privés pendant plusieurs mois. Plus de la moitié d'entre eux regardent également au moins une fois par semaine une vidéo, ce qui en fait le groupe qui compte le plus d'adeptes de ce type de contenu. Ils constituent également la catégorie d'individus qui regroupe le plus de joueurs intensifs, un quart d'entre eux se livrant à cette activité quotidiennement. Par ailleurs, les screenagers présentent des caractéristiques sociodémographiques relativement proches de celles observées parmi l'ensemble des 15-34 ans. On note néanmoins une légère surreprésentation des individus encore en étude ( 40 \% vs. $35 \%$ parmi l'ensemble des $15-34$ ans) et des hommes ( $55 \%$ vs. $50 \%$ ). En matière de pratiques culturelles et de loisirs, les screenagers sont également proches du profil moyen. 


\section{Les computer-centered}

12 Le deuxième profil rassemble $24 \%$ des $15-34$ ans. Il se caractérise par une relative distance vis-à-vis du petit écran. Faibles consommateurs de télévision (près de la moitié la regarde de manière non quotidienne et la très large majorité la regarde moins de 10 heures par semaine), ils ne l'allument pas systématiquement en rentrant chez eux et ils y sont peu attachés (s'ils ne pouvaient plus la regarder, cela leur manquerait peu ou pas du tout). Peu amateurs de vidéos et de jeux vidéo, ils utilisent en revanche quotidiennement l'ordinateur et Internet. Les computer-centered comptent une grande majorité de personnes ayant un niveau de certification scolaire au moins égal au baccalauréat (60\% vs. $42 \%$ des 15-34 ans). Les plus diplômés y sont en nombre ( $22 \%$ de Bac +4 et plus vs. $11 \%$ ). D'origine sociale et culturelle souvent favorisée ( $33 \%$ ont un père cadre ou chef d'entreprise vs. $19 \% ; 31 \%$ ont au moins un parent diplômé du supérieur vs. $18 \%$ ), ils vivent dans un environnement plutôt confortable (53 \% évoluent au sein d'un foyer dont le «chef» est responsable d'entreprise, cadre ou de profession intermédiaire vs. $35 \%$; $24 \%$ vivent dans un foyer au niveau de vie situé dans la tranche supérieure vs. $18 \%$ ) et principalement en milieu urbain (63\% habitent dans une agglomération de plus de 100000 habitants, dans l'agglomération parisienne ou Paris intra-muros vs. $53 \%)$. Les computers-centeredsont assez proches des screenagers en termes d'intensité d'écoute de musique et de fréquence de sorties. S'agissant de la lecture de livres, ils comptent proportionnellement un peu plus de lecteurs modérés à forts.

\section{Les moderates}

De taille équivalente au groupe des computer-centered ( $23 \%$ des $15-34$ ans), les moderates entretiennent, pour leur part, une relation mesurée aux écrans. S'ils regardent quotidiennement le petit écran, leur consommation (en termes de durée) reste cependant dans la moyenne, tout comme celles concernant les vidéos et les jeux vidéo. Par ailleurs, bien qu'utilisant tous l'ordinateur et Internet, ils ne le font pas de manière quotidienne et sont ainsi les moins assidus parmi les utilisateurs de l'informatique. Les moderates rassemblent $21 \%$ de $15-17$ ans (vs. $15 \%$ au sein des 15-34 ans) et forment en cela le groupe le plus jeune. Ils sont peu diplômés ( $69 \%$ n'ont pas le baccalauréat vs. $58 \%$ ) et lorsqu'ils ne suivent plus d'études, les deux tiers d'entre eux sont employés ou ouvriers (vs. $53 \%$ parmi l'ensemble des 15-34 ans ayant terminé leurs études). La majorité des moderates est issue des classes populaires (55\% ont un père ouvrier ou employé vs. $49 \%$; seuls $13 \%$ d'entre eux ont un parent diplômé du supérieur vs. $18 \%$; $39 \%$ d'entre eux vivent dans un foyer dont le " chef de famille » est ouvrier vs. $32 \%$ ) et il s'agit du groupe comptant la part la plus importante d'individus habitant dans une commune rurale ( $25 \%$ vs. $19 \%$ ). Conformément à la moyenne, les moderates déclarent à $82 \%$ préférer en matière de loisirs «plutôt des activités qui les amènent à sortir de chez eux que des activités qui peuvent être pratiquées à la maison ». Pourtant cette préférence ne se traduit pas par une fréquence de sortie plus élevée. Les moderates sont par ailleurs relativement peu investis dans l'écoute musicale et la lecture de livres. 


\section{Les TV-centered}

14 Le quatrième profil est nettement plus minoritaire ( $10 \%$ seulement des individus étudiés). La catégorie des TV-centered se caractérise par un investissement marqué pour les contenus télévisés. Consommateurs quotidiens, comme la plupart des 15-34 ans, la très grande majorité des $T V$-centered allume la télévision tous les jours en rentrant chez eux sans connaître le programme et affirme qu'ils ressentiraient un manque évident s'ils en étaient privés. Ce profil compte la part la plus importante de gros consommateurs de télévision en direct. Si leur intérêt marqué pour le petit écran les rapproche des screenagers, ils s'en distinguent par un faible investissement dans les nouveaux écrans, et plus particulièrement par l'absence ou quasi-absence d'usages de l'ordinateur et d'Internet. Les deux tiers d'entre eux n'ont, de facto, pas utilisé l'ordinateur au cours des douze mois précédents l'enquête, et le dernier tiers s'en est servi moins d'une fois par mois. La catégorie des TV-centered se compose majoritairement de femmes (58 \% vs. $50 \%$ des 15-34 ans). Avec $67 \%$ de 25-34 ans (vs. 51 \%), il s'agit du groupe le plus élevé en âge. Ils ont pour la quasi-totalité quitté le monde des études (seuls $8 \%$ sont encore en étude vs. $35 \%$ ) et sont plus souvent sans emploi ( 47 \% de chômeurs ou d'inactifs vs. 23 \%, avec une part importante de femmes au foyer). Les TV-centered constituent, de loin, le groupe le moins diplômé ( $85 \%$ n'ont pas le baccalauréat vs. $58 \%$; 36 \% n'ont aucun diplôme vs. $16 \%)$. Lorsqu'ils sont sur le marché du travail, ils sont principalement ouvriers ou employés. Ils ont pour la plupart quitté le domicile parental ( $21 \%$ seulement habitent encore chez leurs parents vs. $39 \%$ ) et vivent le plus souvent en couple avec enfant(s) ( $38 \%$ vs. $29 \%$ ), mais les personnes élevant seules leur enfant ne sont pas rares (15\% vs. $5 \%)$. La moitié des TV-centered évolue dans un foyer dont le " chef de famille " est ouvrier (vs. $32 \%$ ) et leurs ressources économiques sont faibles ( $46 \%$ appartiennent à la tranche de revenus par unité de consommation inférieure vs. $19 \%$ ). D'origine sociale et culturelle modeste ( $65 \%$ ont des parents ouvriers ou employés vs. $49 \%$; $70 \%$ des parents n'ayant pas le baccalauréat vs. $57 \%$ ), il s'agit du groupe qui épouse le plus les caractéristiques des classes populaires (Olivier Schwartz (2002) a bien montré cette appétence pour la télévision au sein des classes populaires, phénomène qui selon lui relève la privatisation du monde ouvrier). En matières de pratiques culturelles et de loisirs, les TV-centered constituent le profil qui se distingue le plus: il s'agit notamment du groupe le moins consommateur de musique et comptant également le plus de non-lecteurs de livres (un sur deux contre un sur quatre en moyenne parmi l'ensemble des 15-34 ans). En matière de sorties, si les deux tiers des TV-centered déclarent " préférer plutôt des activités qui les amènent à sortir de chez eux que des activités qui peuvent être pratiquées à la maison ", cette proportion s'avère nettement inférieure à celle observée parmi les autres catégories. Cette attitude casanière se reflète dans leur rythme de sortie, les TV-centered constituant le profil qui sort le moins.

\section{Les no-TV}

Enfin, le profil des no-TV est plus marginal. Il regroupe seulement $3 \%$ des $15-34$ ans $^{6}$ dont la caractéristique la plus distinctive est le non-usage de la télévision. Ils sont également peu adeptes des jeux vidéo et une part non négligeable d'entre eux ne regarde jamais ou presque de contenus vidéo. En revanche, Internet fait partie intégrante de leur quotidien et c'est dans ce profil que la part de gros consommateurs d'ordinateur est la plus élevée : 
la moitié des no-TV passe ainsi au moins 15 heures par semaine devant un ordinateur contre un quart seulement parmi l'ensemble des 15-34 ans. Socialement, les no-TV se situent à l'opposé du groupe des TV-centered. Parmi les cinq profils identifiés, il s'agit de la catégorie qui compte le plus de titulaires du baccalauréat ( $71 \%$ vs. $42 \%$ des $15-34$ ans) et surtout d'individus ayant un niveau de certification scolaire au moins équivalent à Bac+4 ( $34 \%$ vs. $11 \%$ ). La moitié d'entre eux suit encore des études (vs. $35 \%$ ), essentiellement dans le supérieur. Ils ont pour la plupart quitté le domicile parental et vivent le plus souvent seuls et sans enfant ( $31 \%$ vs. $11 \%$ ). Ils bénéficient le plus souvent d'un niveau de vie confortable (supérieur à celui des computer-centered) : le « chef de famille » est le plus souvent cadre ou responsable d'entreprise ( $38 \%$ vs. $16 \%$ ) et les niveaux de vie élevés y sont surreprésentés ( $26 \%$ appartiennent à la tranche supérieure vs. $18 \%$ ). Les habitants de l'agglomération parisienne, et plus encore les habitants de Paris intra-muros, y sont largement surreprésentés ( $22 \%$ sont parisiens vs. $4 \%$ ). Il s'agit du groupe qui compte la proportion la plus importante d'individus issus des classes supérieures ( $49 \%$ ont un parent diplômé du supérieur vs. $18 \% ; 44 \%$ ont un père cadre ou chef d'entreprise vs. $19 \%)$. Les no-TV constituent le groupe le plus investi dans l'écoute de musique. S'ils comptent une proportion d'individus écoutant quotidiennement de la musique comparable à celle observée parmi les profils screenagers et computer-centered, ils sont, en revanche, plus nombreux à mettre quotidiennement de la musique en rentrant chez eux ou bien à écouter de la musique pour elle-même sans rien faire d'autre. Par ailleurs, il s'agit du groupe qui compte le moins de non lecteurs de livres et la part la plus importante de gros lecteurs. En matière de rythme de sorties, les no-TV sont très largement en tête, comme le montre la figure 1.

Figure 1 : Fréquence de sortie selon le profil d'usagers des écrans

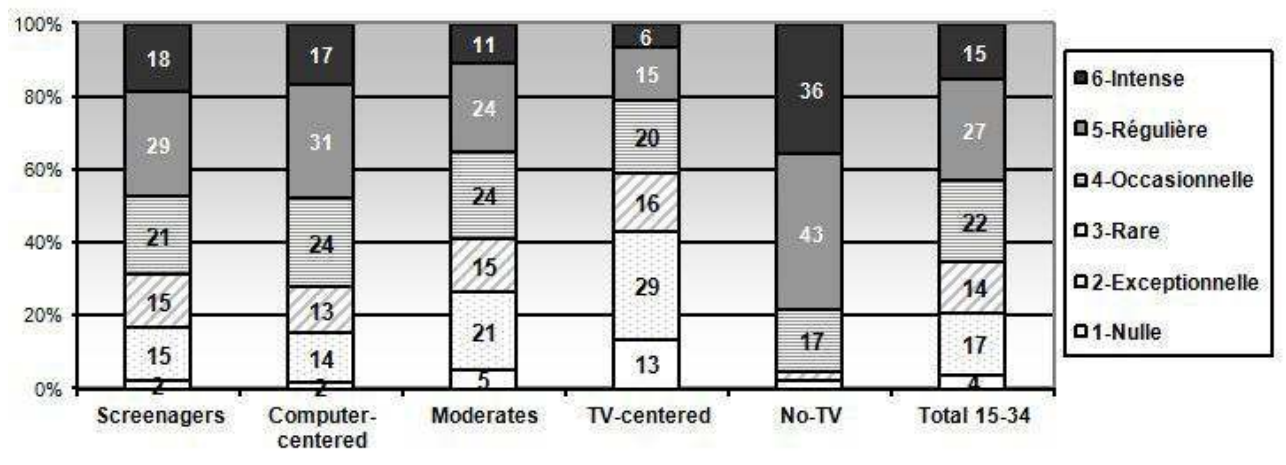

Champs : 15 à 34 ans

Source : enquête Pratiques culturelles 2008, DEPS, ministère de la Culture et de la Communication. Traitement des auteurs.

16 La figure 2 permet de comparer rapidement les différents profils selon le temps moyen hebdomadaire que les jeunes consacrent à l'ensemble des écrans et à sa répartition entre la consommation «traditionnelle » de la télévision et celle des nouveaux écrans (vidéos sur TV, jeux vidéo sur console et ordinateur). 
Figure 2 : Temps moyen hebdomadaire (en heures) passé devant la télévision et les nouveaux écrans selon le profil d'usagers des écrans

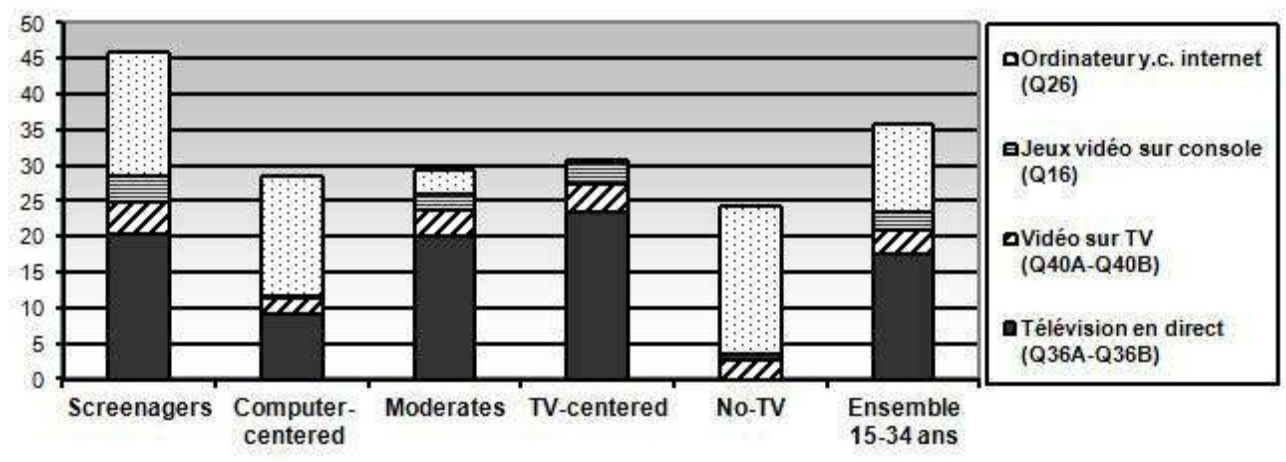

Profils classés du plus au moins répandu : screenagers (39\% des 15-34 ans), computer-centered (24\%), moderates (23\%), TV-centered (10\%), no-TV (3\%)

Champs : 15 à 34 ans

Source : enquête Pratiques culturelles 2008, DEPS, ministère de la Culture et de la Communication. Traitement des auteurs.

Avec 46 heures passées en moyenne chaque semaine devant un écran à titre personnel, les screenagers sont de loin ceux qui consacrent le plus de temps à cette activité, avec une consommation relativement équilibrée entre télévision en direct et nouveaux écrans ( $20 \mathrm{~h}$ vs. 26h par semaine en moyenne). Les screenagers sont par ailleurs les plus gros consommateurs de jeux vidéo sur console et de vidéos sur téléviseur. À l'opposé, les no-TV sont ceux qui consacrent le moins de temps aux écrans avec une moyenne hebdomadaire de 24 heures, quasi uniquement passées devant l'ordinateur. Les trois autres profils ( computer-centered, moderates et TV-centered) passent en moyenne un temps total devant les écrans sensiblement équivalent (autour de 30 heures par semaine), mais avec des répartitions très différentes selon le type d'écran considéré. Moderates et TV-centered investissent principalement la télévision et très peu, voire pas du tout, l'ordinateur, tandis que les computer-centered passent plus de temps devant l'ordinateur que devant le petit écran. Ces derniers sont également les moins consommateurs de jeux vidéo sur console et de vidéos sur téléviseur. Enfin, les no-TV se démarquent surtout par leur nonusage de la télévision traditionnelle. Pour les computer-centered et les no-TV, la relative faiblesse du temps passé à regarder la télévision en direct, à visionner des vidéos sur l'écran de télévision et à se consacrer aux jeux vidéo sur console ne semble pas pour autant compensée par des pratiques accrues de visionnage et de jeux sur ordinateur? ${ }^{7}$.

Cette typologie met d'abord en lumière les usages différenciés des écrans par les jeunes. Toutefois, cette diversité ne saurait être seulement appréhendée comme une série de variations matérielles, lesquelles seraient l'expression des penchants plus ou moins technophiles de la jeunesse et de ses prétendues appétences pour l'éclectisme, voire l'œcuménisme culturels ${ }^{8}$ (Glevarec, 2010b ; 2005 ; Glevarec \& Pinet, 2009). Les différents types d'usage révèlent également des dissemblances pratiques qui sont la traduction d'inégalités sociales et culturelles (Coulangeon, 2011). Les usages des écrans sont le produit d'un ajustement socialement cadré par des goûts, des valeurs, des représentations et des dispositions, lesquels sont inégalement distribués. Ils sont par exemple indexés à des rapports sociaux de sexe qui font des hommes les individus les plus investis dans la pratique : forte présence devant les écrans, valorisation de la culture et 
des loisirs informatiques, individualisation des pratiques, etc., tandis que les femmes semblent moins promptes à s'engager de la sorte (e.g. en s'isolant), préférant les écrans pouvant être plus aisément mobilisés sans avoir nécessairement à déroger aux obligations domestiques chronophages qui leur échoient encore bien souvent et auxquelles elles sont acculturées relativement tôt, surtout dans les milieux populaires : s'occuper des enfants (frères ou sœurs), participer à l'entretien des sociabilités familiales, aider à tenir le domicile, etc. (Bonnafous, Jouët \& Rieffel, 2003 ; van Zoonen, 2002). Comme l'expliquait Bourdieu, «les agents sociaux, et aussi les choses en tant qu'elles sont appropriées par eux, donc constituées comme propriétés, sont situés en un lieu de l'espace social, lieu distinct et distinctif qui peut être caractérisé par la position qu'il occupe par rapports à d'autres lieux » (Bourdieu, 1997 : 161) ; les écrans participent de ces choses.

\section{Les déterminants des attitudes face aux écrans}

19 Afin d'évaluer l'effet propre des différentes dimensions potentiellement explicatives (notamment le capital scolaire, l'origine sociale et le capital économique) sur la pratique des écrans, nous avons modélisé les probabilités d'appartenance à chacun des profils (le profil des screenagers, le plus répandu, étant choisi comme référence) ${ }^{9}$. Dans un premier temps nous avons modélisé ces probabilités d'appartenance par seulement trois dimensions : le genre, l'âge et l'origine sociale (estimée par le diplôme le plus élevé des parents). Ce choix de ne pas introduire d'autres dimensions résulte, d'une part, de l'existence de corrélations quasi-mécaniques entre variables explicatives qui risqueraient de conduire à des erreurs d'interprétation et, d'autre part, du fait qu'un certain nombre de caractéristiques s'interprètent différemment selon l'âge des individus. Par exemple, le capital scolaire, mesuré par le diplôme obtenu le plus élevé, n'a de sens que pour les individus ayant achevé leurs études (ou du moins une bonne partie). Rappelons que $97 \%$ des 15-17 ans sont encore en étude (contre $55 \%$ des 18-24 ans et seulement $4 \%$ des 25-34 ans). Dans un second temps, nous avons considéré chacune des trois tranches d'âge (15-17 ans, 18-24 ans, 25-34 ans) et procédé à des modélisations séparées. Nous avons ainsi cherché à identifier (de manière différenciée selon les classes d'âge envisagées), les éventuels effets propres du genre, du capital scolaire, du capital culturel familial, du capital économique, du statut d'activité et de leur situation familiale ${ }^{10}$.

\section{La primauté de l'origine sociale sur l'âge et le genre}

La figure 3 permet de comparer les répartitions des différents profils selon l'âge, le sexe et l'origine sociale de l'individu (mesurée par le niveau de diplôme le plus élevé des parents). 
Figure 3 : Répartition des profils d'usagers des écrans selon l'âge, le sexe et l'origine sociale (mesurée par le niveau de diplôme le plus élevé des parents)
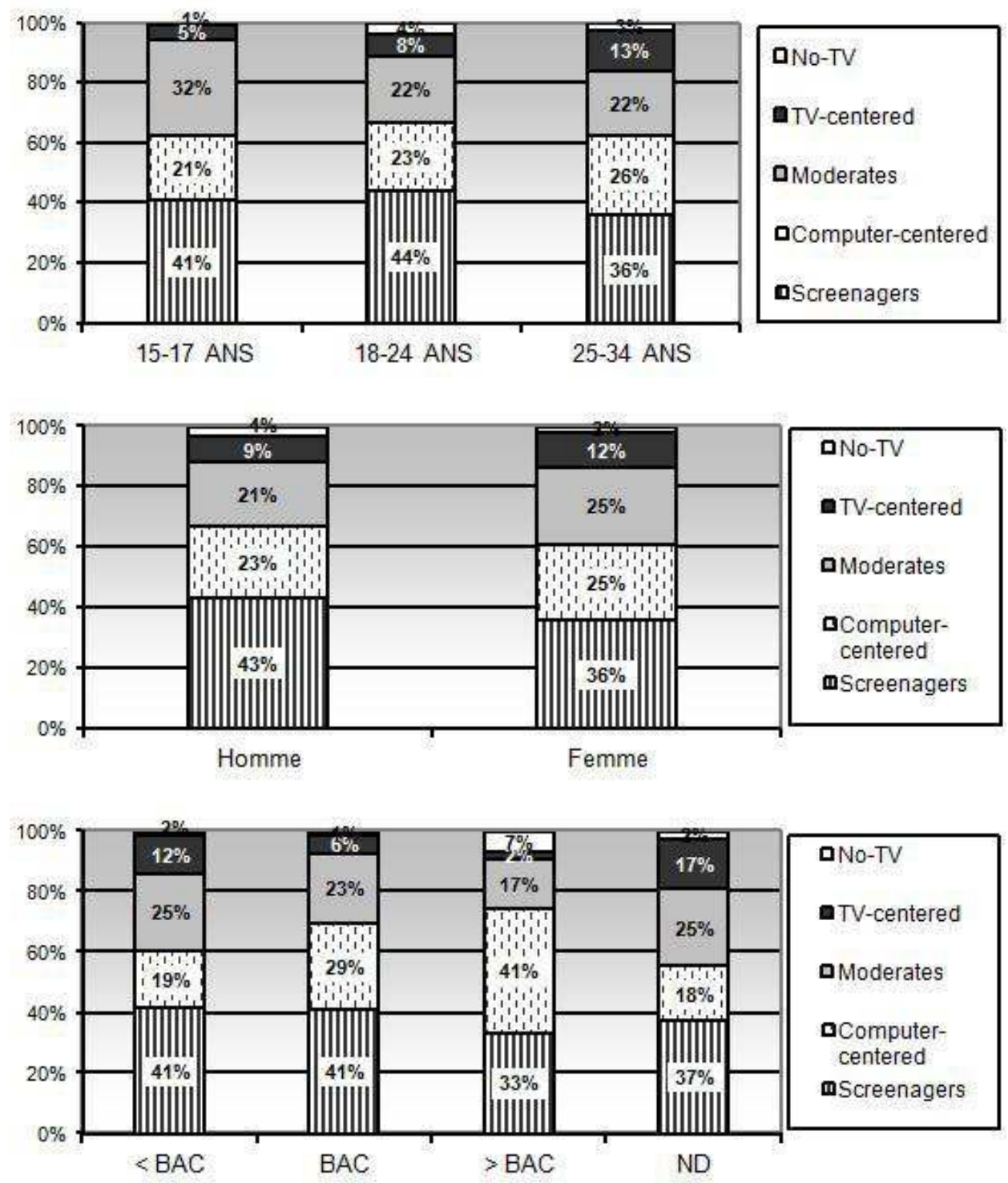

Champs : 15 à 34 ans

Source : enquête Pratiques culturelles 2008, DEPS, ministère de la Culture et de la Communication. Traitement des auteurs.

S'agissant de l'âge d'abord, quelle que soit la tranche considérée, les no-TV, profil dont la caractéristique principale est de ne jamais regarder la télévision, restent marginaux, ne dépassant pas les $5 \%$. Les TV-centered, autrement dit les exclus de l'informatique et d'Internet, voient en revanche leur part augmenter avec l'âge, représentant seulement $5 \%$ des 15-17 ans, mais atteignant $13 \%$ parmi les 25-34 ans. Les screenagers demeurent le profil le plus courant (non majoritaire), pour chaque tranche d'âge considérée, mais il est toutefois moins répandu parmi les plus âgés. Les computer-centered sont présents en proportion relativement similaire dans les trois tranches d'âge. Si les écrans sont massivement mobilisés par les jeunes, leurs usages ne sont pas pour autant homogènes et se déclinent pour partie en fonction des âges de la jeunesse (Octobre, 2009). Notamment, les plus jeunes sont davantage investis dans la manipulation de l'informatique connectée 
que leurs aînés, celle-ci leur permettant de centraliser autour de l'ordinateur un ensemble d'activités de consommation culturelle et ludique, de production de contenus et d'entretien du lien social (blogs, messagerie instantanée, sites de réseaux sociaux, etc.) qui participe d'une certaine « culture de la chambre » (Glevarec, 2010a). Avec l'avancée en âge, les modalités expressives de soi, la construction des rôles sociaux, la recherche d'autonomie vis-à-vis des parents et les formes d'individuation se nuancent, épousent différents contours, s'appuient sur d'autres prises matérielles et trouvent à se ressourcer au sein d'autres contextes sociaux (Metton-Gayon, 2009). Pour autant, le franchissement des étapes propres à la période juvénile, n'explique pas toutes les différences constatées.

La comparaison des répartitions selon le genre révèle une proportion un peu plus importante des profils pas ou peu utilisateurs de l'ordinateur. Ainsi, $37 \%$ des femmes ont un profil TV-centered ou moderates contre $30 \%$ seulement des hommes. Inversement, ces derniers comptent une part un peu plus élevée de screenagers, lesquels investissent plus fortement l'ensemble des différents écrans (TV, ordinateur et consoles de jeux) et les pratiques qui leur sont liées (regarder la télévision, visionner des vidéos, jouer à des jeux vidéo, utiliser l'ordinateur et Internet). La part des computer-centered est en revanche semblable entre les hommes et les femmes. Les travaux de Sylvie Octobre $(2011 \mathrm{a} / \mathrm{b})$ montrent que les usages de la culture sont fortement genrés (chez les 11-18 ans). Si les équipements révèlent des différences notables (e.g. les garçons sont mieux dotés en jeux vidéo et les filles en matériel audio), ce sont aussi les rapports entretenus à la culture et aux écrans qui sont dissemblables. L'écran télévisuel s'avère par exemple particulièrement prisé par une certaine frange de la gent féminine plus attirée par la romance et la fiction, tandis que les usages féminins des autres écrans sont moins orientés par les aspects ludiques et compétitifs et davantage tournés vers des pratiques plus « sérieuses ».

23 Enfin, les répartitions des différents profils diffèrent fortement selon l'origine sociale. Nombre de jeunes n'ayant aucun parent titulaire du baccalauréat sont peu ou pas utilisateurs de l'ordinateur. Ainsi, $38 \%$ d'entre eux ont un profil moderates ou TV-centered contre seulement $19 \%$ des jeunes ayant au moins un parent diplômé de l'enseignement supérieur. Lorsqu'ils investissent l'ordinateur, les jeunes issus d'un milieu faiblement diplômé adoptent davantage le profil screenagers, contrairement aux jeunes issus d'un milieu au capital scolaire élevé qui adoptent plutôt le profil computer-centered. Par ailleurs, si le profil no-TV reste peu représenté quelle que soit l'origine sociale, il n'est toutefois plus tout à fait marginal parmi les jeunes les plus favorisés ( $7 \%$ des $15-34$ ans ayant au moins un parent diplômé de l'enseignement supérieur ont un profil de no-TV). On voit ici combien les phénomènes de socialisation culturelle restent d'une importance capitale. Malgré la massification scolaire, la démocratisation de certaines institutions culturelles, le développement des médias de masse et des industries du loisir, la montée de l'éclectisme, etc., autant de phénomènes qui multiplient les occasions de fréquentation d'une variété toujours plus grande de culturèmes, les legs familiaux forgent des dispositions durables. Celles-ci peuvent perdre de leur force de cadrage au gré des trajectoires biographiques des individus qui en sont porteurs, mais elles continuent cependant à persister, structurant nombre de leurs pratiques présentes, quand bien même celles-ci tendent sensiblement à se déporter vers des dispositifs et des services de consommation plus récents (Coulangeon, $2011: 104)$.

Dans la modélisation destinée à évaluer l'effet propre de ces trois dimensions (âge, genre, origine sociale), la procédure de sélection de variables conduit à introduire tout d'abord 
l'origine sociale. Autrement dit, lorsque l'on considère indépendamment les trois dimensions, l'origine sociale est la plus significative. L'estimation des odds ratios ${ }^{11}$, ou rapports des chances, nous montre qu'à âge et sexe maintenus constants, avoir un parent diplômé de l'enseignement supérieur, plutôt que titulaire du seul baccalauréat, multiplie par plus de 5 les chances d'adopter un profil de no-TV, profil très faiblement représenté et par près de 2 celles d'adopter un profil de computer-centered (relativement au profil screenagers pris pour référence). Inversement, avoir des parents non titulaires du baccalauréat augmente les chances relatives d'être un TV-centered (multiplie par 1,7) et diminue celles d'être un computer-centered (divise par 1,6). Ce qui se révèle ici, à gros traits, c'est sans doute le signe d'une forme particulière de la stratification sociale des (dé)goûts. Sans doute peut-on faire l'hypothèse que la moindre appétence pour l'univers télévisuel permet de se distinguer de ceux qui épousent les goûts les plus mainstream de la culture de masse, lesquels autorisent soit l'affirmation de son appartenance à la majorité, soit une forme de singularisation par adhésion à certaines sous-cultures (Pasquier, 2005). L'univers de l'informatique connectée, sans constituer un écosystème techno-culturel inhabituel, se voit néanmoins affecté d'un quotient de rareté supérieur à celui de l'univers télévisuel et peut, de ce fait, servir de support de distinction, notamment à ceux pour qui l'usage de l'informatique connectée fait rupture avec les habitudes culturelles de leur milieu d'origine. Les écrans de la néo-télématique se trouvent, selon toute vraisemblance, dans une situation carrefour où les pratiques plastiques qu'ils supportent et les manières dont celles-ci font sens pour les utilisateurs relèvent d'investissement assez variés. Les (non-)usages se développent sous la contraintes de ressources inégalement distribuées, mais ils sont également le support de projections symboliques divergentes, leur prêtant des capacités et des vertus dissemblables dans l'ordre de la culture : avoir la possibilité d'accéder à des éléments de culture moins standardisés pour les uns ou, au contraire, de fréquenter des culturèmes de la culture de masse pour les autres, tout en mobilisant des manières de faire qui les différencient, par exemple, de leurs pairs TV-centered et ainsi avoir l'impression d'échapper aux affres de l'indignité.

Par ailleurs, l'estimation de l'effet propre de l'âge nous montre qu'avoir entre 15 et 17 ans (plutôt qu'entre 18 et 24 ans) augmente les chances relatives d'être un moderate (multiplie par 1,6), tandis qu'avoir entre 25 et 34 ans augmente celles d'être un computer-centered (multiplie par 1.7) ou d'un TV-centered (multiplie par 1,9). Enfin, être une femme plutôt qu'un homme augmente les chances relatives d'adopter le profil TV-centered (multiplie par 1,6), moderates (multiplie par 1,4) et computer-centered (multiplie par 1,3) plutôt que screenagers. Les modélisations menées séparément sur les trois tranches d'âges (15-17, 18-24 et 25-34 ans) nous permettent de préciser les effets des dimensions explicatives ${ }^{12}$.

\section{À l'adolescence (15-17 ans)}

Pour les grands adolescents (15-17 ans), seul le genre a un effet significatif (au seuil de $5 \%$ ) sur la probabilité d'appartenance aux différents profils d'attitude face aux écrans. En effet, si la totalité ou presque des 15-17 ans sont à la fois consommateurs de télévision, d'ordinateur, de vidéos et de jeux vidéo ${ }^{13}$, le poids des différents profils d'usagers des écrans varie selon le sexe (Figure 4). 
Figure 4 : Répartition des profils d'usages des écrans selon le genre chez les 15-17 ans.



Champs : 15 à 17 ans

Source : enquête Pratiques culturelles 2008, DEPS, ministère de la Culture et de la Communication. Traitement des auteurs.

Ainsi, les garçons adoptent davantage un profil de screenagers (48 \% des garçons de 15-17 ans vs. 33 \% des filles du même âge). La moindre prégnance des screenagers (profil le plus consommateur d'écrans) parmi les adolescentes peut s'expliquer par des contraintes temporelles de loisirs plus importantes chez les filles qui s'impliquent davantage dans leur scolarité et déclarent plus souvent que leurs camarades masculins avoir trop de devoirs et manquer de temps pour leurs loisirs (Céroux \& Crépin, 2011). Le temps dédié aux écrans serait ainsi limité par le temps consacré aux devoirs, ainsi qu'aux loisirs scolairement rentables, comme la lecture. Par ailleurs, si la pratique des jeux vidéo s'est largement diffusée depuis la fin des années 1990, quelle que soit la tranche d'âge considérée, Olivier Donnat montre que «le caractère traditionnellement masculin de cette activité ne s'est pas effacé au cours de la dernière décennie, particulièrement si l'on écarte les joueurs occasionnels. [...] L'écart est même d'une plus grande ampleur dans les générations les plus investies dans cette activité » (Donnat, 2009 : 39 ; Octobre et al., 2010). D'une manière générale, les activités reposant sur une forte composante technique (photographie, vidéo, informatique, etc.) sont moins investies par les filles, même si celles-ci ont aujourd'hui plus largement pris leur part au sein des publics les plus technophiles et tendent ainsi à féminiser certaines pratiques qui étaient jusqu'alors quasi "réservées » à la gent masculine (Octobre, 2011a). Une fois introduit le genre dans le modèle, les autres dimensions testées (diplôme, capital culturel et économique familial) ne sont pas significatives (au seuil de $5 \%$ ). Mais n'oublions pas que les effectifs sont ici limités (seuls 213 individus sont âgés de 15 à 17 ans).

\section{Aux premières années de l'âge adulte (18-24 ans)}

Si un effet de genre a été mis en évidence chez les 15-17 ans, il n'est en revanche plus observé chez les 18-24 ans. Seul le statut d'activité, et plus précisément le fait de suivre des études, a un impact significatif sur la probabilité d'appartenir aux différents profils. En effet, les 18-24 ans qui poursuivent des études utilisent tous l'ordinateur (moins de $1 \%$ appartient aux TV-centered), alors que le non-usage de l'ordinateur n'est pas marginal parmi les individus de la même classe d'âge occupant un emploi ou bien étant sans activité (respectivement $13 \%$ et $19 \%$ de TV-centered). Les moderates, utilisateurs non quotidiens de l'ordinateur et d'Internet, sont également peu répandus parmi les 
étudiants, ce qui n'est pas le cas parmi les actifs occupés et les chômeurs ou inactifs. Ainsi, un jeune sur deux sorti du système éducatif n'utilise pas l'ordinateur (TV-centered) ou en fait un usage non quotidien (moderates). Inversement, l'omniprésence des deux profils utilisant l'informatique quotidiennement, computer-centered et screenagers, parmi les jeunes poursuivant des études montre combien l'ordinateur est devenu incontournable dans la vie étudiante et combien le fait d'effectuer des études longues incite à fréquenter l'univers de l'informatique connectée, lequel tend vraisemblablement à devenir une ressource culturelle durable. Ce résultat confirme ce que nous avions pu remarquer dans une enquête précédente (Granjon, 2009a), qui mettait notamment en avant que la fraction la plus diplômée des classes populaires développaient des usages important de l'informatique connectée, y compris après que leurs études aient été terminées. Nous avions également souligné que l'intensité de ces pratiques était supérieure à celle qu'il était possible de constater au sein des classes moyennes et supérieures. Par ailleurs, si le profil des screenagers reste le plus répandu parmi les étudiants, notons qu'il est seulement 1,2 fois plus répandu que le profil des computercentered, contre 2,6 fois parmi les actifs occupés et 3,8 parmi les chômeurs et inactifs. Enfin, si une fois pris en compte le fait de poursuivre des études, l'origine sociale n'a pas d'effet significatif sur la probabilité d'appartenance aux différents profils d'usagers des écrans, il ne faudrait pas conclure trop rapidement à la neutralité de l'origine sociale. Rappelons qu'outre le fait de disposer d'effectifs relativement limités (528 individus âgés de 18 à 24 ans), la poursuite d'études après 18 ans est fortement corrélée au niveau de diplôme des parents : $37 \%$ des 18-24 ans en étude ont au moins un parent diplômé de l'enseignement supérieur contre seulement $12 \%$ des actifs occupés et $8 \%$ des chômeurs ou inactifs.

Figure 5 : Répartition des profils d'usages des écrans selon le statut d'activité chez les 18-24 ans

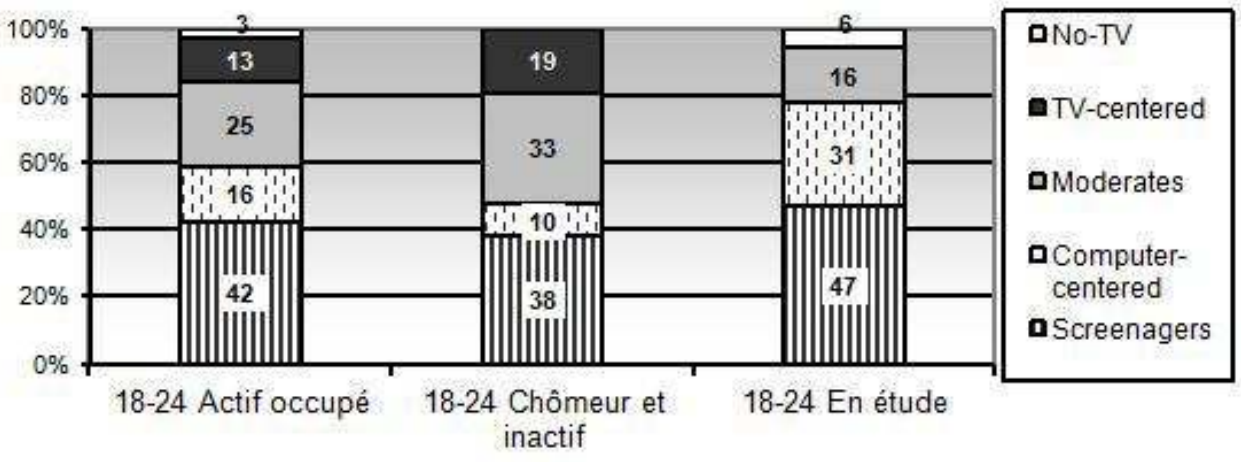

Champs : 18 à 24 ans

Source : enquête Pratiques culturelles 2008, DEPS, ministère de la Culture et de la Communication. Traitement des auteurs.

\section{À l'âge de l'indépendance (25-34 ans)}

Enfin, pour les 25-34 ans, la modélisation met en évidence un effet propre du capital scolaire, du capital économique, du genre et de l'origine sociale sur l'appartenance aux différents profils d'usage des écrans. Toutes choses égales par ailleurs (c'est-à-dire à capital économique, genre et capital scolaire maintenus constants), être issu d'un milieu peu diplômé (aucun des parents n'ayant le baccalauréat, plutôt qu'avoir au moins un de 
ses parents titulaire au plus de celui-ci) réduit les chances d'être un computer-centered plutôt qu'un screenager. Être une femme augmente les chances d'avoir un profil de moderates ou de TV-centered plutôt que de screenagers. Pour les 25-34 ans, l'effet genre joue de manière différente de celui observé parmi les 15-17 ans. Si l'ordinateur est devenu un outil qui rassemble peu ou prou les filles et les garçons de 15-17 ans, ce n'est pas tout à fait le cas de la tranche d'âge 25-34 ans, pour laquelle une partie des femmes restent en retrait, l'ordinateur conservant encore, en partie, le caractère masculin du loisir technique (Bonnafous, Jouët \& Rieffel, 2003). Ainsi, $17 \%$ des femmes ayant entre 25 et 34 ans sont des TV-centered contre seulement $9 \%$ des hommes du même âge. Outre le fait d'être une femme, être non ou peu diplômé (plutôt qu'avoir uniquement le baccalauréat) et disposer d'un faible capital économique (niveau de vie dans la tranche inférieure) multiplie les chances d'appartenir aux TV Fans plutôt qu'aux screenagers.

\section{Conclusion}

La dernière enquête Pratiques culturelles des Français montre combien les écrans ont envahi la sphère des loisirs de l'ensemble de la population. Début 2008, ceux-ci sont devenus incontournables avec seulement $1 \%$ des Français n'utilisant ni ordinateur, ni télévision. Si pour les plus âgés (les plus de 65 ans) l'usage des écrans se résume quasi exclusivement au visionnage de programmes en direct sur le petit écran, complété pour certains par le visionnage occasionnel de vidéos sur leur téléviseur, la consommation d'écrans s'est en revanche largement diversifiée chez les moins de 35 ans. Ces derniers apparaissent en effet comme des utilisateurs très concernés par les écrans et plus particulièrement encore par les nouveaux écrans, même si l'intensité de leurs usages dessine un paysage nuancé. Comme le rappelle Sylvie Octobre (2009), les 11-18 ans font notamment montre d'un fort niveau de connexion, d'une importante assiduité à l'informatique connectée, d'une appétence marquée pour les usages tournés vers la communication et d'un goût avéré pour certains loisirs comme les jeux vidéo, le téléchargement ou encore la production de contenus. Mais en observant de plus près les fréquences de pratiques (regarder la télévision, des vidéos, jouer à des jeux vidéo, utiliser l'ordinateur et Internet) et le temps consacrés aux différents écrans (téléviseur, ordinateur, console de jeux), les jeunes sont loin de tous se retrouver sous un profil homogène :

La notion de digital natives recouvre donc des réalités à géométrie fort variable, entre jeunes d'âge, d'origine sociale et de sexe différents, le poids de chacune de ces variables se combinant pour composer des rapports à la culture formant un continuum de situations allant des plus impliqués et éclectiques aux exclus, en passant par des mono-passionnés d'une consommation ou d'une activité. Les jeunes générations présentent d'ailleurs probablement un plus large spectre de rapports à la culture que leurs aînées, celle-ci entrant dans un dispositif de représentation de soi tout à fait central chez elles. (Octobre, 2009)

31 Ainsi, la figure du jeune fan des écrans, boulimique de télévision, de vidéos, de jeux vidéo, d'informatique et d'Internet, que nous avons décrite sous l'appellation de screenagers est, certes, la catégorie d'utilisateurs la plus répandue au sein de la jeunesse, mais elle ne concerne néanmoins que quatre jeunes sur dix. Il ne faut notamment pas oublier qu'un tiers des jeunes n'utilise pas ou peu l'ordinateur. Les cinq profils d'usagers des écrans ici identifiés, construits uniquement en fonction de leurs pratiques et de leurs fréquences d'usages, et donc sans tenir compte des types de contenus mobilisés ni des goûts déclarés, apparaissent déjà fortement clivés socialement. Les no-TV présentent les attributs des 
classes les plus favorisées, tandis que les TV-centered arborent les attributs des classes populaires, suivis de près par les moderates. L'étude des déterminants des profils d'usagers des écrans nous montre alors la primauté de l'origine sociale sur l'âge et le genre quant à la probabilité d'adopter le profil de no-TV ou celui de computer-centered (profils non ou peu consommateurs de télévision, de vidéos et de jeux vidéo). Ce que montrent les usages des écrans c'est la pérennité de la composante culturelle des inégalités sociales, laquelle n'est pas soluble dans les discours qui font des technologies de l'information et de la communication les nouveaux fétiches de la «société du savoir» et de l'idéologie de la communication (Granjon, 2009b). Si les homologies structurales mises au jour dans La Distinction (Bourdieu, 1979), liant positions dans l'espace social, consommations culturelles et styles de vie ne sont certainement plus aussi prégnantes aujourd'hui, elles n'en disparaissent pas pour autant, tant s'en faut. De nombreux travaux montrent au contraire que les inégalités sociales sont toujours à l'œuvre, d'une autre manière, mais continuent bel et bien à cadrer les pratiques culturelles, fussent-elles numériques (Bennet et al., 2009). L'importance prise par les variables «sexe/genre » et " âge », et dont il est évidemment important de reconnaître qu'elles jouent un rôle essentiel dans la morphologie des usages des écrans, ne doit cependant pas faire oublier que pour comprendre les dynamiques culturelles générationnelles, il faut garder en vue que ces critères tombent également sous le coup de rapports sociaux qui en configurent les effets spécifiques (Pfefferkorn, 2007; Chauvel, 2002). Sylvie Octobre constate par exemple que chez les moins de 18 ans

l'origine sociale clive plus durablement les rapports des enfants des deux sexes dans les milieux populaires à cet objet [l'ordinateur] catégorisé "masculin". Chez les enfants de cadres, ajoute-t-elle, l'avantage initial des garçons ne dure pas puisque ces derniers sont rattrapés par leurs homologues filles en seconde moitié de collège et au lycée. Ce rattrapage n'a en revanche pas lieu dans les milieux populaires, où les garçons sont à tous âges plus investis dans l'usage de l'ordinateur que les filles, les enfants de cadres ayant par ailleurs toujours un niveau de pratique supérieur aux enfants d'ouvriers (Octobre, 2011b : 33).

La jeunesse ne décrit donc pas une période du cycle de vie où la pesanteur des déterminations sociales serait momentanément levée et les différenciations de type "sexe-genre/âge » qui s'y constatent doivent être mises en relation avec les autres facteurs producteurs d'inégalités sociales.

L'unité apparente des classes d'âge s'avère de facto traversée par des réalités sociales dissemblables qui en difractent l'uniformité. Et si les matériaux de l'enquête EPCF 2008 ici traités permettent d'apporter un premier éclairage sur les différentes cultures d'écrans des jeunes, il est évident que, d'une part, les analyses que nous en avons proposées sont loin d'épuiser la richesse des données disponibles et que, d'autre part, il est essentiel de s'appuyer sur des appareils de preuve complémentaires, notamment qualitatifs, afin de cerner, au plus près des pratiques effectives et des contenus mobilisés, les différentes formes d'appropriation des écrans par la jeunesse. De fait, les enquêtes qualitatives que nous avons menées, mais dont nous n'avons pu ici rendre compte, permettent de restituer plus finement les usages des écrans. Elles tendent notamment à confirmer que, selon leur appartenance sociale, les utilisateurs ne saisissent pas les mêmes attributs décisifs des écrans et n'en développent pas les mêmes usages. Les usages sociaux des dispositifs audiovisuels, informatiques et télématiques s'avèrent ainsi liés aux appréciations, envies, intérêts, goûts et au sens pratique de ceux qui les mobilisent. Ils sont le résultat d'un ajustement complexe entre une histoire sociale incorporée (les schèmes 
de perception et d'action des usagers) et la mobilisation d'un artefact technique qui est lui-même constitué d'une combinatoire de mondes sociaux et culturels. Les réalités empiriques des usages sociaux des écrans se différencient donc d'un groupe social à un autre. Les capacités pratiques des utilisateurs sont marquées par leur appartenance de classe, tout en étant évidemment travaillées par la forte variété de leurs parcours biographiques.

\section{BIBLIOGRAPHIE}

BENNET Tony, SAVAGE Mike, siLva Elizabeth, WARDE Alan, GAYo-CAL Modesto \& WRIGHT David (2009). Culture, Class, Distinction, London/New York, Routledge.

BONNAFOUS, Simone, JoUËT, Josiane \& RIEFFEL, Rémy (dir.) (2003). " Une communication sexuée ?", Réseaux, 21 (120).

BOURDIEU Pierre (1979). La Distinction. Critique sociale du jugement, Paris, Minuit.

BOURDIEU Pierre (1984). « La jeunesse n'est qu'un mot », in Questions de sociologie, Paris, Minuit, pp. 143-154.

BOURDIEU Pierre (1997). Méditations pascaliennes, Paris, Seuil.

CÉROUX Benoît \& CRÉPIN Christiane (2011). Les relations entre les parents et leurs enfants à l'aune des loisirs des adolescents, Direction des Statistiques, des Études et de la Recherche, CAF, dossier d'études, 140.

CHAUVEL Louis (2002). Le destin des générations : structure sociale et cohortes en France au XX⿳亠丷⿵冂丶 ${ }^{2}$ siècle, Paris, PUF.

COULANGEON Philippe (2011). Les métamorphoses de la distinction. Inégalités culturelles dans la France d'aujourd'hui, Paris, Grasset.

DONNAT Olivier (2009). Les pratiques culturelles des Français à l'ère numérique. Enquête 2008, Paris, La Découverte.

DONNAT Olivier \& LÉVY Florence (2007). Approche générationnelle des pratiques culturelles et médiatiques, Paris, DEPS, Ministère de la Culture et de la Communication.

GIRE Fabienne, PASQUIER Dominique \& GRANJON Fabien (2007). « Culture et sociabilité. Les pratiques de loisirs des Français », Réseaux, 25 (145-146), pp. 160-215.

GLEVAREC Hervé (2005). « La fin du modèle classique de la légitimité culturelle. Hétérogénéisation des ordres de légitimité et régime contemporain de justice culturelle. L'exemple du champ musical » in Éric Maigret \& Éric Macé (dir.), Penser les médiacultures. Nouvelles pratiques et nouvelles approches de la représentation du monde, Paris, Armand Colin/Ina, pp. 69-102.

GLEVAREC Hervé (2010a). La culture de la chambre. Pré-adolescence et culture contemporaine dans l'espace familial, Paris, La Documentation française.

GLEVAREC Hervé (2010b). « La tablature des genres culturels appliquée à la télévision », Les Cahiers de la SFSIC, 5, pp. 18-19. 
GLEVAREC Hervé \& PINET Michel (2009). « La "tablature” des goûts musicaux : un modèle de structuration des préférences et des jugements ", Revue française de sociologie, 50 (3), pp. 599-640. GRANJON Fabien (2009a). « Les usages du PC au sein des classes populaires. Inégalités numériques et rapports sociaux de classe, de sexe et d'âge », in Fabien Granjon, Benoit Lelong \& Jean-Luc Metzger (dir.), Inégalités numériques. Clivages sociaux et modes d'appropriation des TIC, Paris, Hermès/ Lavoisier, pp. 31-62.

GRANJON Fabien (2009b). «Inégalités numériques et reconnaissance sociale. Des usages populaires de l'informatique connectée », Les Cahiers du numérique, 5 (1), pp. 19-45.

GRANJON Fabien (2010). « Le « non-usage » de l'Internet : reconnaissance, mépris et idéologie », Questions de communication, 18, pp. 37-61.

GRANJON Fabien \& COMBES Clément (2007). « La numérimorphose des pratiques de consommation musicale. Le cas de jeunes amateurs », Réseaux, 25 (145-146), pp. 292-334.

GRANJON Fabien, COMBES Clément \& VILLE Sandrine (2009). Les régimes de consommation des contenus audiovisuels, rapport Orange Labs, Issy-les-Moulineaux, 45 pages.

METTON-GAYON Céline (2009). Les adolescents, leur téléphone et Internet. Tu viens sur MSN ?, Paris, L'Harmattan

остовRE Sylvie (2009). «Pratiques culturelles chez les jeunes et institutions de transmission : un choc des cultures », Culture prospective, 1 .

остовRE Sylvie (2011a). « Présentation. Le genre, la culture et l'enfance », Réseaux, 29 (168-169), pp. 9-22.

остовRE Sylvie (2011b). « Du féminin et du masculin. Genre et trajectoires culturelles », Réseaux, 29 (168-169), pp. 23-57.

oCTOBRe Sylvie, DÉTREZ Christine, MERCKLÉ Pierre \& BERTHOMIER Nathalie (2010). L'enfance des loisirs. Trajectoires communes et parcours individuels de la fin de l'enfance à la grande adolescence, Paris, La Documentation française.

PASQUIER Dominique (2005). Cultures lycéennes. La tyrannie de la majorité, Paris, Autrement.

PFEFFERKORN Roland (2007). Inégalités et rapports sociaux. Rapport de classes, rapports de sexe, Paris, La Dispute.

SCHWARTZ Olivier (2002). Le monde privé des ouvriers, Paris, PUF.

VAN ZOONEN Liesbet (2002). «Gendering the Internet. Claims, Controversies and Cultures ", European Journal of Communication, 17 (1), pp. 5-23.

\section{ANNEXES}

\section{Annexe 1 - Construction de l'indicateur de revenu du foyer par unité de consommation}

Afin de comparer les niveaux de vie des individus, nous avons créé une nouvelle variable ramenant le revenu du foyer par unité de consommation. 
Nous avons considéré l'ensemble des enquêtés (et pas uniquement les 15-34 ans) afin que l'indicateur puisse être utilisé pour d'autres analyses.

Comme le revenu est un revenu déclaré en tranches, nous avons tout d'abord estimé le revenu par le milieu de la classe et nous avons arbitrairement fixé le revenu des classes extrêmes (500 euros pour « 609 euros ou moins par mois » et 7500 euros pour « plus de 6099 euros par mois »).

Nous avons alors calculé le nombre d'unités de consommation du foyer pour chaque enquêté en utilisant la définition disponible sur le site de l'INSEE.

Unité de consommation

Système de pondération attribuant un coefficient à chaque membre du ménage et permettant de comparer les niveaux de vie de ménages de tailles ou de compositions différentes. Avec cette pondération, le nombre de personnes est ramené à un nombre d'unités de consommation (UC).

Pour comparer le niveau de vie des ménages, on ne peut s'en tenir à la consommation par personne. En effet, les besoins d'un ménage ne s'accroissent pas en stricte proportion de sa taille. Lorsque plusieurs personnes vivent ensemble, il n'est pas nécessaire de multiplier tous les biens de consommation (en particulier, les biens de consommation durables) par le nombre de personnes pour garder le même niveau de vie.

Aussi, pour comparer les niveaux de vie de ménages de taille ou de composition différente, on utilise une mesure du revenu corrigé par unité de consommation à l'aide d'une échelle d'équivalence. L'échelle actuellement la plus utilisée (dite de l'OCDE) retient la pondération suivante :

-1 UC pour le premier adulte du ménage ;

$-0,5$ UC pour les autres personnes de 14 ans ou plus ;

$-0,3$ UC pour les enfants de moins de 14 ans $^{14}$.

Nous avons alors divisé le revenu par unité de consommation et déterminé quatre tranches correspondant aux quartiles sur la population ayant déclaré son revenu (19\% de refus de réponse ou de «ne se prononce pas »-nsp).

Le tableau suivant présente la répartition de l'ensemble des Français de plus de 15 ans selon le revenu du foyer par unité de consommation et la répartition obtenue sur la souspopulation des 15-34 ans. La tranche 1 correspond au niveau de vie le plus bas et la tranche 4 au niveau de vie le plus haut.

Tableau 2 : Répartition des Français selon le revenu du foyer par unité de consommation (en 4 tranches)

\begin{tabular}{|l|l|l|}
\hline & Ensemble des français de 15 ans et + & Ensemble des Français de 15 à $\mathbf{3 4}$ ans \\
\hline Refus ou nsp & $19 \%$ & $22 \%$ \\
\hline Tranche $\mathbf{1}$ & $18 \%$ & $19 \%$ \\
\hline Tranche 2 & $22 \%$ & $21 \%$ \\
\hline Tranche 3 & $20 \%$ & $19 \%$ \\
\hline
\end{tabular}




\begin{tabular}{|l|l|l|}
\hline Tranche 4 & $21 \%$ & $19 \%$ \\
\hline Total & $100 \%$ & $100 \%$ \\
\hline
\end{tabular}

\section{Annexe 2 - Modélisation des probabilités d'appartenir à chaque profil d'usages des écrans selon le l'âge, le sexe et l'origine sociale}

Tableau 3 : Estimation des odds ratios du modèle logit multinomial avec sélection de variables (stepwise) - probabilités d'appartenir à chaque profil d'usages des écrans comparativement au profil des screenagers

\begin{tabular}{|c|c|c|c|c|c|}
\hline \multicolumn{2}{|c|}{ Modalité de référence : screenagers } & $\begin{array}{l}\text { Computer- } \\
\text { centered }\end{array}$ & Moderates & $\begin{array}{l}\text { TV- } \\
\text { centered }\end{array}$ & No-TV \\
\hline \multicolumn{2}{|c|}{$\begin{array}{l}\text { Part effective parmi les } 15-34 \text { ans } \\
\text { (screenagers : } 39 \% \text { ) }\end{array}$} & $24 \%$ & $23 \%$ & $10 \%$ & $3 \%$ \\
\hline \multirow{4}{*}{$\begin{array}{l}\text { Diplôme le plus } \\
\text { élevé des parents }\end{array}$} & $<$ BAC & $0.626^{* *}(1)$ & n.s & $1.726^{*}$ & n.s \\
\hline & $B A C$ & Réf. & Réf. & Réf. & Réf. \\
\hline & $>$ BAC & $1.852 * * *$ & n.s & n.s & $\begin{array}{l}5.545 \\
* * *\end{array}$ \\
\hline & $\begin{array}{l}\text { Nsp, père et mère } \\
\text { inconnus, refus }\end{array}$ & n.s & n.s & $2.429 * *$ & n.s \\
\hline \multirow{3}{*}{ Âge } & $15-17$ ans & n.s & $1.603 * *$ & n.s & n.s \\
\hline & 18-24 ans & Réf. & Réf. & Réf. & Réf. \\
\hline & $25-34$ ans & $1.684^{* * *}$ & n.s & $1.895 * * *$ & n.s \\
\hline \multirow{2}{*}{ Sexe } & Homme & Réf. & Réf. & Réf. & Réf. \\
\hline & Femme & $1.330 * *$ & $1.441 * * *$ & $1.646^{* * *}$ & n.s \\
\hline
\end{tabular}

Lecture : *** significatif au seuil de $1 \%$, ** significatif au seuil de $5 \%$, significatif au seuil de $10 \%$, n.s non significatif à $10 \%$. Les modalités de référence sont signalées par réf. Les odds ratios, ou rapports des chances, représentent les variations de probabilité d'appartenir à chacun des profils d'usagers des écrans par rapport au profil de référence (screenagers) pour une variation d'une des caractéristiques des dimensions explicatives retenues. (1) Ainsi, pour les 15-34 ans, à sexe et âge donné, avoir des parents non titulaires du bac divise par 1.597 (=1/0.626) les chances d'appartenir au profil computercentered plutôt qu'au profil screenagers.

Source : enquête Pratiques culturelles 2008, DEPS, ministère de la Culture et de la Communication. Traitement des auteurs. 


\section{Annexe 3 - Modélisation des probabilités d'appartenir à chaque profil d'usages des écrans - Traitement séparé selon l'âge de l'individu}

Les résultats des trois modélisations (un modèle pour chaque tranche d'âge considérée) sont présentés dans le tableau 3.

Pour les grands adolescents (15-17 ans), nous cherchons à identifier les éventuels effets propres du genre, du diplôme (être titulaire du BEPC-CAP-BEP vs. aucun diplôme), du capital familial, culturel (approché par le diplôme le plus élevé des parents) et économique (PCS du chef de famille, le niveau de revenu du foyer étant ignoré ou non déclaré par près de la moitié des 15-17 ans).

Pour les jeunes adultes (18-24 ans), nous cherchons à identifier les éventuels effets propres du genre, de leur capital scolaire (selon deux modalités : être titulaire ou non du bac), du statut d'activité (selon trois modalités : poursuivre des études, occuper un emploi, être sans activité c'est-à-dire chômeur ou inactif), de l'origine sociale (approchée par le diplôme le plus élevé des parents), du capital économique (CSP du chef de famille puisque le niveau de revenu du foyer est souvent ignoré ou non déclaré par les 18-24 ans) et de leur situation familiale (selon cinq modalités : vivre chez ses parents, en couple sans enfant, en couple avec enfant, élève seule son enfant, célibataire sans enfant).

Pour les 25-34 ans, nous cherchons à identifier les éventuels effets propres du genre, de leur capital scolaire (selon trois modalités : sans le bac, titulaire du bac, diplômé du supérieur), du statut d'activité (selon trois modalités : poursuivre des études, occuper un emploi, être sans activité c'est-à-dire chômeur ou inactif), de l'origine sociale (approchée par le diplôme le plus élevé des parents), du capital économique (selon le niveau de vie, c'est-à-dire le revenu du foyer par unité de consommation, en quatre tranches) et de leur situation familiale (selon cinq modalités : vivre chez ses parents, en couple sans enfant, en couple avec enfant, élève seule son enfant, célibataire sans enfant).

Remarque : si contrairement à la modélisation effectuée sur l'ensemble des 15-34 ans, les trois modélisations séparées ne font apparaître aucune dimension explicative significative (au seuil de $5 \%$ ) sur le rapport des chances d'appartenir au profil des no-TV, ce résultat doit être pris avec précaution, le profil étant très faiblement répandu et les effectifs limités.

Remarque : si contrairement à la modélisation effectuée sur l'ensemble des 15-34 ans, les trois modélisations séparées ne font apparaître aucune dimension explicative significative (au seuil de $5 \%$ ) sur le rapport des chances d'appartenir au profil des no-TV, ce résultat doit être pris avec précaution, le profil étant très faiblement répandu et les effectifs limités.

Tableau 4 : Estimation des odds ratios des modèles logit multinomial avec sélection de variables (stepwise) - probabilités d'appartenir à chaque profil d'usages des écrans comparativement au profil des screenagers - traitement séparé selon l'âge

\begin{tabular}{|l|l|l|l|l|}
\hline $\begin{array}{l}\text { Modalité de référence : } \\
\text { screenagers }\end{array}$ & $\begin{array}{l}\text { Computer- } \\
\text { centered }\end{array}$ & Moderates & $\begin{array}{l}\text { TV- } \\
\text { centered }\end{array}$ & $\begin{array}{l}\text { No- } \\
\text { TV }\end{array}$ \\
\hline
\end{tabular}




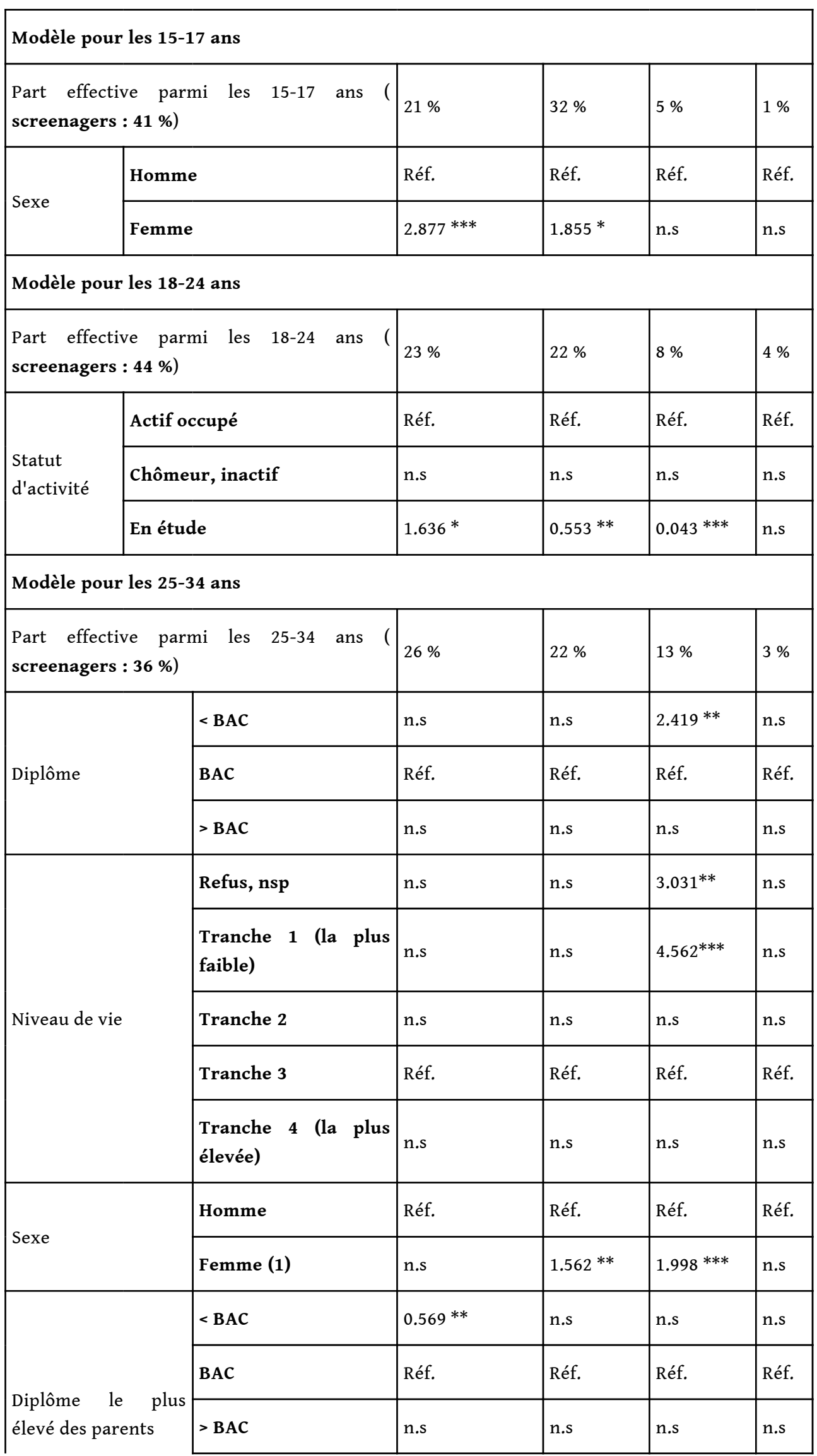




\begin{tabular}{|l|l|l|l|l|l|}
\hline $\begin{array}{l}\text { Nsp, père et mère } \\
\text { inconnus, refus }\end{array}$ & $0.389 * *$ & n.s & n.s & n.s \\
\hline
\end{tabular}

Les variables non significatives au seuil de $5 \%$ de sont pas introduite dans le modèle.


non significatif à $10 \%$. Les modalités de référence sont signalées par réf. Les odds ratios, ou rapports des chances, représentent les variations de probabilité d'appartenir à chacun des profils d'usagers des écrans par rapport au profil de référence (screenagers) pour une variation d'une des caractéristiques des dimensions explicatives retenues. (1) Ainsi, pour les 25-34 ans, à autres caractéristiques introduites dans le modèle maintenues constantes, être une femme multiplie par près de 2 les chances d'appartenir au profil TV-centered plutôt qu'au profil screenagers.

Source : enquête Pratiques culturelles 2008, DEPS, ministère de la Culture et de la Communication. Traitement des auteurs.

\section{NOTES}

1. Ce large périmètre englobe l'ensemble des classes d'âge au-delà desquelles on constate un décrochage important dans l'intensité des usages des écrans et notamment dans celle de l'ordinateur. À l'évidence, entre 15 ans et 34 ans, les sujets sociaux traversent des situations très contrastées au regard du cycle de vie, lequel décrit des évolutions relevant à la fois de l'avancée en âge, du statut familial et du statut professionnel. Pour conduire certaines analyses, il s'avère donc nécessaire de réintroduire des distinctions plus fines liées à l'âge, ce que nous ne manquerons pas de faire par la suite.

2. Le travail statistique au fondement de cet article a été effectué à partir des données de l'enquête Pratiques culturelles des français réalisée par le ministère de la culture en 2008. Pour la première fois depuis sa création, elle intègre un ensemble de questions portant sur les pratiques numériques. Nous remercions chaleureusement Olivier Donnat de bien avoir voulu partager ces précieux matériaux d'enquête, soit les réponses apportées par 5004 individus de 15 ans et plus résidant en France, interrogés en face à face entre le 29 novembre 2007 et le 10 février 2008. Les analyses portent plus spécifiquement sur un sous-échantillon de 1577 individus âgés de 15 à 34 ans.

3. Le premier axe factoriel, qui contient 7,6\% de l'inertie totale, est construit sur une corrélation mécanique entre les 4 variables traduisant l'intensité d'usage et l'attachement à la télévision (la même modalité "ne regarde jamais ou pratiquement la télévision » est répétée dans chaque variable). Comme le premier axe factoriel, le deuxième axe qui contient 5,4 \% de l'inertie totale, est principalement construit sur une corrélation quasi-mécanique entre les trois variables relatives à l'usage de l'ordinateur. Ainsi les non-utilisateurs de l'ordinateur s'opposent aux utilisateurs réguliers (principalement quotidiens). Le troisième axe représente $4,7 \%$ de l'inertie totale et le quatrième axe $4 \%$.

4. À l'instar de l'enquête sur les pratiques culturelles des Français (EPCF 2008), nous désignons comme lecteurs modérés les individus qui déclarent avoir lu entre 10 et 19 livres au cours des 12 mois précédant l'enquête. Au-delà de 20 livres, nous les considérons comme de forts lecteurs.

5. Mais cette modalité passe juste le critère de la valeur test et, au surplus, il s'agit d'une modalité rare, ce qui limite la portée du résultat.

6. Seuls 44 individus relèvent de ce profil.

7. Regarder sur l'ordinateur des films, séries ou vidéos téléchargés, des DVD loués, achetés ou gravés, jouer sur ordinateur à des jeux vidéo achetés ou téléchargés, à des jeux en réseau, utiliser Internet pour regarder en direct un programme de télévision ne sont pas plus répandus (voire moins) parmi les computer-centered et les no-TV que parmi les screenagers. 
8. Hervé Glevarec et Michel Pinet (2009) remettent par exemple en cause l'existence d'une hiérarchie culturelle qu'ils considèrent comme un pur "vice de forme", dans la mesure où, affirment-ils, les sociologues (critiques) seraient les témoins aveuglés de l'incommensurabilité des contenus culturels. Selon eux, les phénomènes sociaux d'usages distinctifs de contenus culturels seraient en voie d'épuisement, laissant place à un régime contemporain de justice culturelle ne permettant plus de consacrer des différences de valeurs entre genres culturels, mais signalant une disposition à l'ouverture et à la tolérance, laquelle redéfinirait le jugement social entre amateurs et goûts. Il n'y aurait évidemment plus d'homologies structurales entre le social et le culturel, mais plus encore, le fait même d'évoquer des profils omnivores, éclectiques ou dissonants seraient une aberration témoignant d'une allégeance dépassée aux théories de la légitimité qui considèrent les genres culturels comme des marqueurs sociaux.

9. Il s'agit d'un modèle de régression logistique (modèle logit multinomial), avec sélection des variables introduites dans le modèle par la méthode stepwise.

10. L'ensemble des modélisations sont présentées en annexe.

11. L'estimation des odds ratios est présentée dans l'annexe 2.

12. Les trois modélisations sont présentées dans l'annexe 3.

13. Rappelons que $1 \%$ seulement des $15-17$ ans ne regarde jamais la télévision, $3 \%$ n'ont pas d'usages personnels de l'ordinateur, $4 \%$ n'utilisent pas Internet, $5 \%$ ne regardent jamais ou presque des vidéos et $10 \%$ ne jouent pas aux jeux vidéo.

14. Source : Insee, consulté le 24 octobre 2011.

\section{RÉSUMÉS}

Les enquêtes sur les pratiques culturelles des français montrent combien les pratiques numériques font désormais partie des loisirs de la population, en particulier de sa frange la plus jeune. Loin, cependant, de la vision souvent idéalisée d'une génération de « digital natives » toute convertie aux nouveaux écrans, les données révèlent des écarts de pratiques considérables entre les plus jeunes. Cet article propose, à partir du traitement secondaire des données de l'Enquête pratiques culturelles des français de 2008, de montrer la diversité des pratiques des 15-34 ans. L'analyse factorielle permet de produire une typologie en cinq catégories, screenagers, TV-centered , moderates, no-TV et computer-centered, et la recherche des déterminants de ces catégories révèle le rôle différencié du genre, de l'âge et de l'origine sociale. Ce dernier facteur reste premier dans l'explication des pratiques.

Surveys of cultural practices among the French population show how much digital practices are now part of people's leisure time, especially among the youngest. However, far from the idealized picture of a generation of 'digital natives', of which every member would be an adept of new screens, data reveal considerable gaps among the youngest people. Based on the secondary analysis of data from the 2008 French national survey on cultural practices (Enquete pratiques culturelles des français), this article attempts to show the diversity of practices among the 15-34 year old people. Five types ('screenagers', 'TV-centered', 'moderates', 'no-TV' and 'computercentered') emerge through factor analysis and the search for their determinants reveals the differentiated roles of genre, age and social origin, this last factor remaining primary in the explanation of practices. 
INDEX

Mots-clés : jeunes, loisirs, numérique, genre, origine sociale

Keywords : youth, leisure, digital, gender, social origin

\section{AUTEURS}

\section{FABIENNE GIRE}

Orange Labs, SENSE

\section{FABIEN GRANJON}

Université Paris 8 Vincennes - Saint-Denis, CEMTI 